\title{
La prescription de placebo, question éthique délicate
}

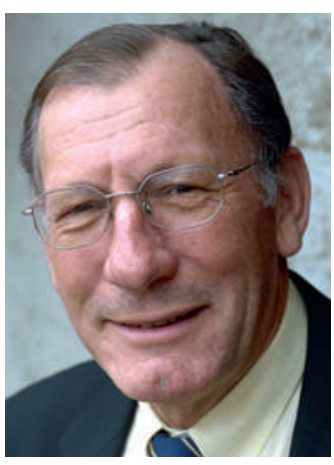

Jean Martin

\section{Références}

1 AMA. Code of Medical Ethics, Opinion 8.083: Placebo Use in Clinical Practice. 2006.

2 Barnhill A. Clinical use of placebos: Still the physician's prerogative? Hastings Center Report. 2012;42 (3):29-37.

3 Kolber A. A limited defense of clinical placebo deception. Yale Law and Policy Review. 2007-8;26;75-134

4 Foddy B. A duty to deceive: placebos in clinical practice. American Journal of Bioethics. 2009;9 (12):4-12.

* L'Académie suisse des sciences médicales a tenu en novembre 2009, avec la Faculté de médecine de Zurich, une réunion sur le thème

«Placebointerventionen im ärztlichen Alltag»: www.samw.ch/Projekte/ Archiv/Placebointerventionen-im-aerztlichen-Alltag. html

Et l'Institut d'éthique biomédicale de Zurich organise à fin août 2012 à Ascona un symposium "Beyond the placebo»: http://www.ethik.uzh.ch/ ibme/veranstaltungen/ placebo.html
Depuis une génération, l'émergence des droits des patients a mis l'accent sur l'exigence d'une information préalable, compréhensible et suffisamment complète du malade sur les observations du médecin et les examens et thérapeutiques qu'il propose. La question du placebo prend ainsi une nouvelle dimension.

Le Code éthique de l'Association médicale américaine (AMA) interdit aux médecins de prescrire des substances dont ils pensent qu'elles sont des placebos, à moins que le patient soit informé et accepte [1]. Un article récent du Hastings Center Report [2] discute la différence à faire entre deux doctrines: celle qui demande aux médecins de se référer au consensus professionnel sur ce qui est un placebo ou pas, d'une part, et d'autre part celle qui qualifie comme placebos seulement les substances ou démarches que le médecin traitant considère lui-même comme tels. En d'autres termes: si le praticien administre une substance vue comme placebo par la connaissance médicale majoritaire mais que lui-même estime qu'elle a une réelle efficacité, alors il ne s'agirait pas (déontologiquement) d'un traitement par placebo. Cette seconde conception correspond à l'avis de l'AMA, que Barnhill critique.

Exemple majeur: l'homéopathie est considérée comme de nature placebo par beaucoup de médecins allopathes mais ce n'est évidemment pas le cas pour ceux qui la pratiquent. A propos d'acupuncture et d'une manière qui surprend, Barnhill en parle comme si elle était encore souvent vue comme un placebo alors que, pour le traitement de la douleur en particulier, il est admis aujourd'hui qu'elle a une valeur.

Extraits de l'opinion émise en 2006 par l'AMA: «Les médecins peuvent utiliser des placebos dans un but de diagnostic ou de traitement seulement si le patient est informé et accepte son usage. Un placebo peut être efficace même si le patient sait qu'il sera utilisé mais qu'il n'est pas en mesure de l'identifier (...) De cette façon, le médecin respecte l'autonomie du patient et promeut une relation de confiance.» «Permettre aux médecins de transmettre leur propres opinions positives sur une méthode sans faire état du scepticisme majoritaire dans la profession peut améliorer la réceptivité des patients et l'efficacité du traitement, mais cela peut aussi menacer l'autonomie du patient.»

Ces situations incluent une importante zone grise entre des extrêmes en noir et blanc: du médecin qui délibérément fait une prescription qu'il sait inef- ficace à celui qui prescrit un médicament courant dans une indication qui n'est pas scientifiquement démontrée. A noter que prescrire un antibiotique pour une affection virale est donner un placebo. Ample continuum...

La notion de placebo ne concerne pas des produits seulement mais d'autres techniques, notamment du domaine des méthodes alternatives. Avec toujours cette différence que certains jugeront qu'il s'agit de purs placebos agissant par autosuggestion alors que ceux qui les proposent sont convaincus de leur efficacité: ainsi des techniques de yoga, relaxation, méditation. Les anciens parmi nous se souviennent du «traitement» des verrues en faisant tourner à vide un appareil de radiologie... D'importance actuelle, la question de la chirurgie placebo: pour qu'un traitement chirurgical soit prouvé efficace, il convient d'un point de vue EBM de le comparer avec la sham surgery (où on incise sans intervenir sur le fond). Dans ce dernier cas, il est clair que le consentement informé préalable du patient est impératif.

En général, on pense ici à la formule qui dit que ce qui guérit a raison («Wer heilt, hat recht» en allemand). Formule que refusent vigoureusement ceux qui affirment que la médecine fondée sur les preuves est l'entier de la vérité du soin et qu'il ne peut pas y avoir de contribution utile de choses que la «Schulmedizin» n'a pas légitimé... Mais il faut garder à l'esprit qu'on ne saurait affirmer qu'une technique est inefficace simplement parce qu'on n'en connaît pas (encore) le mécanisme d'action.

Certains bioéthiciens estiment que donner des placebos sans informer les patients peut être éthique quand c'est le meilleur traitement disponible [3, 4]. Cela peut être le cas quand la médecine scientifique a atteint ses limites: si un malade n'est pas soulagé par un traitement d'efficacité prouvée ou si le traitement actif donne lieu à des effets secondaires intolérables, tenter l'usage de placebos est une alternative admissible.

«Les médecins peuvent éviter d'employer des placebos et pourtant produire un effet placebo par l'usage compétent de réassurance, d'encouragement et de good bedside manner» [2]. Ce qui met le doigt sur le fait que, dans la pratique quotidienne, même si le médecin a pour principe de ne pas en prescrire il est souvent lui-même/elle-même le placebo.*

Jean Martin, membre de la rédaction et de la Commission nationale d'éthique 